\title{
Acetabular distraction hip arthroplasty in neglected transverse and T-shape acetabular fractures : a different application
}

\author{
Ayman Abdelaziz Bassiony, Saleh Gameel, Ahmed Nageeb Mahmoud
}

From the Ain Shams University Hospitals, Cairo, Egypt

\begin{abstract}
Neglected acetabular fractures are often challenging to treat. The aim of this study is to highlight the technique of acetabular distraction with porous metal cup as a viable technique to address such complex cases.

This is a prospective study of seven patients who had neglected acetabular fracture or fracture non-union that were managed with total hip arthroplasty using acetabular distraction and porous metal cup with cemented liner.

The average age of the patients was 51 years (range 36-58 years). Average time of follow up was 18 months (range 12-36 months). Radiological results at latest follow up and by comparing serial radiographs showed that all cases had a well-fixed implant construct without evidence of loosening or malorientation. Average Harris hip score pre-operatively was 46, which improved to an average of 84 post-operatively. Our study shows that total hip replacement using acetabular distraction with porous metal cup provide early good results in reconstruction of acetabular bony deficiencies due to neglected acetabular fracture.
\end{abstract}

Keywords : Acetabular fracture non-union ; pelvic discontinuity; acetabular distraction; total hip arthroplasty.

\section{INTRODUCTION}

Acetabular fractures are often serious injuries which can lead to progressive impairment of hip function and patient quality of life. Anatomic reduction with rigid internal fixation is the ideal management to restore the hip function and prevent long-term complications. Unfortunately, many patients with fractures of the acetabulum still suffer posttraumatic arthritis or femoral head necrosis regardless of whether operative or non-operative intervention was chosen as the initial treatment (1).

On the other hand, neglected acetabular fractures are defined as fractures that present more than three weeks after injury (2). Although these cases are rare, they can be still found especially in developing countries (3). With expected higher incidence of complications if surgical intervention is delayed (4), an established acetabular fracture non-union for example reportedly does not heal and often progresses through rapid osteoarthritic changes (4).

The role of primary Total hip arthroplasty (THA) in acetabular fractures is controversial. Damage to femoral head and difficult reconstructions of

Ayman Abdelaziz Bassiony, MD, Professor of orthopedic surgery

- Saleh Gameel, MD, Lecturer of orthopedic surgery

- Ahmed Nageeb Mahmoud, MD, Lecturer of orthopedic surgery Ain Shams University, Cairo, Egypt

Correspondence : Ayman Abdelaziz Bassiony, Orthopedic Department, Ain Shams University, Cairo, Egypt. Mobile : 01001625333.

Email : aymanbassiony@yahoo.com

- 2021, Acta Orthopædica Belgica. 
the acetabulum are quoted as ideal indications. However, stability of the cup may be inadequate in such a situation leading to early failure (5). On the other hand, in neglected and poorly treated cases, THA is usually offered as a reliable procedure in the surgical management $(5,6)$.

Acetabular distraction technique, first described in detail by Sporer et al (7), demonstrated encouraging clinical and radiographic outcomes in reconstructing cases with chronic pelvic discontinuity due to acetabular defects. Since then, there has been few reports with respect to its use revision hip arthroplasty (8-10) and however, to our knowledge, no reports documented about its use in complex primary hip arthroplasty following neglected or complicated acetabular fracture cases. The aim of this study is to describe the surgical technique and early clinical outcomes of distraction arthroplasty using porous metal acetabular shell in treating patients with neglected acetabular fractures as a new indication.

\section{PATIENTS AND METHODS}

This is a prospective case series including 7 patients who had neglected acetabular fractures, in whom clinical and radiological decision of total hip replacement and acetabular reconstruction was made. Patients were 5 males and 2 females, with a mean age of 51 years (range 36-58 years) and with at least three-months old and significantly displaced acetabular fractures. Established radiological fracture non-union, in the form of obvious interfragmentary gaps and local bone defects was recognized in all cases and femoral head protrusion in 2 cases. The average time from acetabular fracture to the hip replacement operation was 6 months (range 3-12 months). The delay in the presentation was due to either patient unfitness for definitive surgery during the acute setting, lack of the medical service in rural locations, or patients'/ surgeons' choice of conservative management. The patients were followed in regular intervals ( 2 weeks, 6 weeks, 3, 8, 12 month and annually for a mean of 18 months (range 12-36 months). None of the patients was lost in follow up (Table 1).

Preoperatively, acetabular bone deficiency and fracture pattern were assessed based on Anteroposterior and lateral view $\mathrm{X}$ rays supplemented by CT scan. At first laboratory investigations in the form of complete blood picture, ESR and CRP were done to rule out infection.

The patient is placed and held in the lateral decubitus position. Special care is needed to make sure that the patient's pelvis is perpendicular to the table, to facilitate the identification of anatomical landmarks and orientation of the acetabulum. A posterolateral approach was utilized in all cases because it allows for excellent exposure of both the acetabular columns, ilium, and the femur. The sciatic nerve was identified during the approach and protected.

Prior to performing acetabular distraction, the integrity of the anterosuperior and posteroinferior columns must be evaluated to identify any large defects of either column that may require tantalum augment reconstruction or bone graft. After achievement of adequate acetabular visualization, any acetabular floor fibrous membrane and fibrous

Table 1. - Patients' demographics, diagnosis, the size of the acetabular component used and the follow up period.

\begin{tabular}{|c|c|c|c|c|c|}
\hline Case & $\begin{array}{c}\text { Age (Years) } \\
\text { and Sex }\end{array}$ & $\begin{array}{c}\text { Time to surgery } \\
\text { (Months) }\end{array}$ & Diagnosis & $\begin{array}{c}\text { Size of the inserted } \\
\text { Tantalum shell }\end{array}$ & FU \\
\hline 1 & $36 \mathrm{M}$ & 7 & Transverse fracture & $60 \mathrm{~mm}$ & 12 \\
\hline 2 & $54 \mathrm{~F}$ & 12 & Transverse fracture & $64 \mathrm{~mm}$ & 12 \\
\hline 3 & $49 \mathrm{M}$ & 7 & Transverse fracture & $60 \mathrm{~mm}$ & 14 \\
\hline 4 & $52 \mathrm{M}$ & 5 & Transverse and posterior wall fracture & $64 \mathrm{~mm}$ & 18 \\
\hline 5 & $58 \mathrm{M}$ & 3 & Transverse fracture & $60 \mathrm{~mm}$ & 18 \\
\hline 6 & $52 \mathrm{M}$ & 4 & Transverse and posterior wall fracture & $60 \mathrm{~mm}$ & 36 \\
\hline 7 & $56 \mathrm{~F}$ & 4 & T. fracture & & \\
\hline
\end{tabular}


callus is carefully curetted, and bone loss is assessed. Cobb elevator is used to stress the inferior aspect of the ischium.

A Schanz screw is placed in the ischium and the superior dome. A large laminar spreader is then placed over the screws allowing for distraction from an extra-articular position (Fig. 1). Sequential reaming of the bony walls is then carefully performed to reshape the acetabular walls in order achieve an adequate line to line fit with the trial acetabular cup. Prior to implantation of the final acetabular shell, crushed cancellous bone autograft is placed into the acetabular cavity and impacted into any remaining defect, and then reamed on reverse to force the graft into the created acetabular walls (Fig 1).

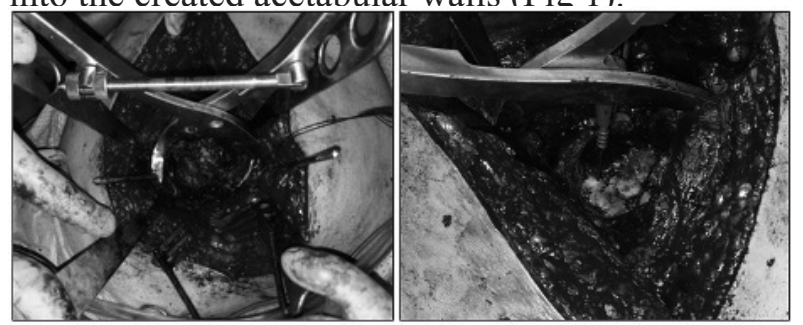

Figure 1. - Application of the acetabular distractor and cancellous autograft.

The acetabular component chosen (Zimmer Trabecular Metal Shell ${ }^{\circledR}$, Warsaw, IN) for implantation was the same size as the last reamer. The correct cup size should catch in the acetabulum but also comfortably disengage. Through the acetabular cup, multiple cancellous screws are placed into the remaining ilium and ischium. With this largediameter, porous metal acetabular component acting as an "internal plate", the discontinuity or the fracture is bridged and treated in distraction. (Fig 2).

The distracting force is then released, and the pelvis is allowed to recoil under ligamentotaxis to further secure the construct. The liner is finally cemented into place, using Palacos ${ }^{\circledR}$ antibiotic loaded cement (Heraeus, Germany) with optimal liner alignment (Fig 3).

On the day after surgery, physical therapy is initiated with transfer training along with active motion exercises for the hip and strengthening activities. Weight bearing on the affected limb was restricted for 6 weeks, and then partial weight

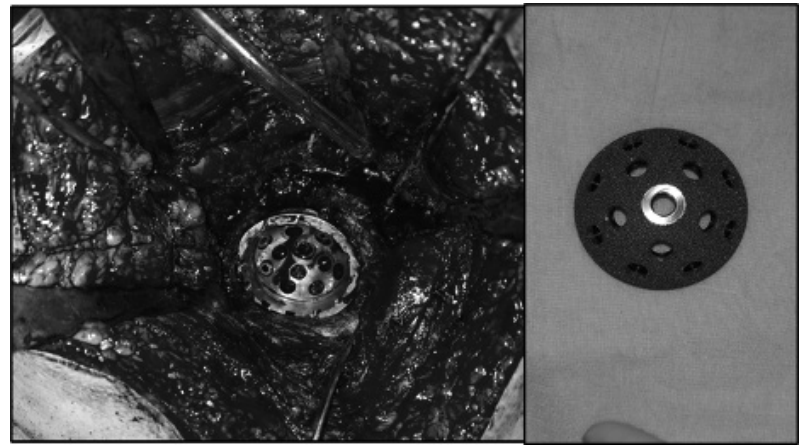

Figure 2.- The final configuration of the shell after releasing distraction. The tantalum cup was applied and fixed with multiple screws to stabilize both acetabular columns before the distraction on the two Schanz screws is released.

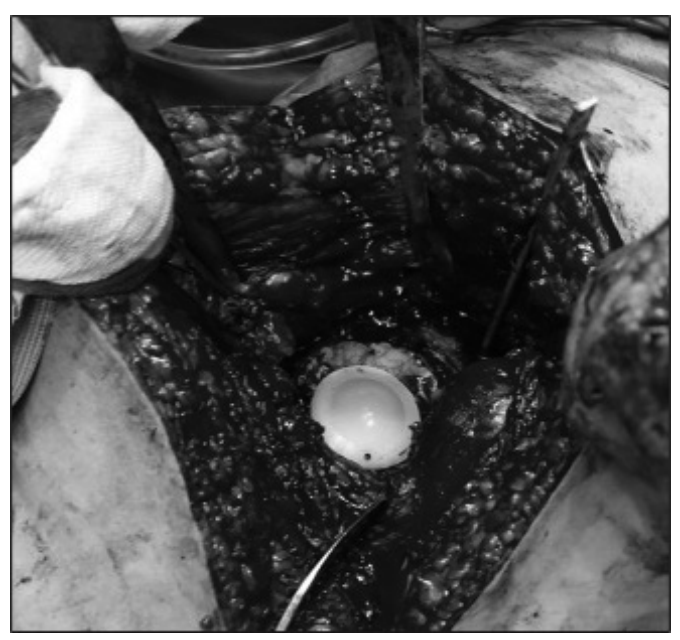

Figure 3. - Highly cross-linked polyethylene cup is cemented in place over the tantalum cup.

bearing was allowed, progressively starting from toe touch, for the following 6 weeks. Routine deep vein thrombosis prophylaxis with an oral anticoagulant is prescribed until partial weight bearing starts.

Clinical evaluations were performed at all followup intervals using the Harris hip score (HHS) (6). A score of 90 to 100 was considered as excellent, 80 to 90 as good, 70 to 80 as fair, and below 70 as poor. Success of revision was defined as an increase in the scores of 20 or more points, a stable cup, with no additional surgery on the acetabulum (11).

Radiological evaluation was done through anteroposterior and lateral radiographs at regular followup intervals (Fig 4). Radiolucent lines adjacent to the acetabular component were identified as 

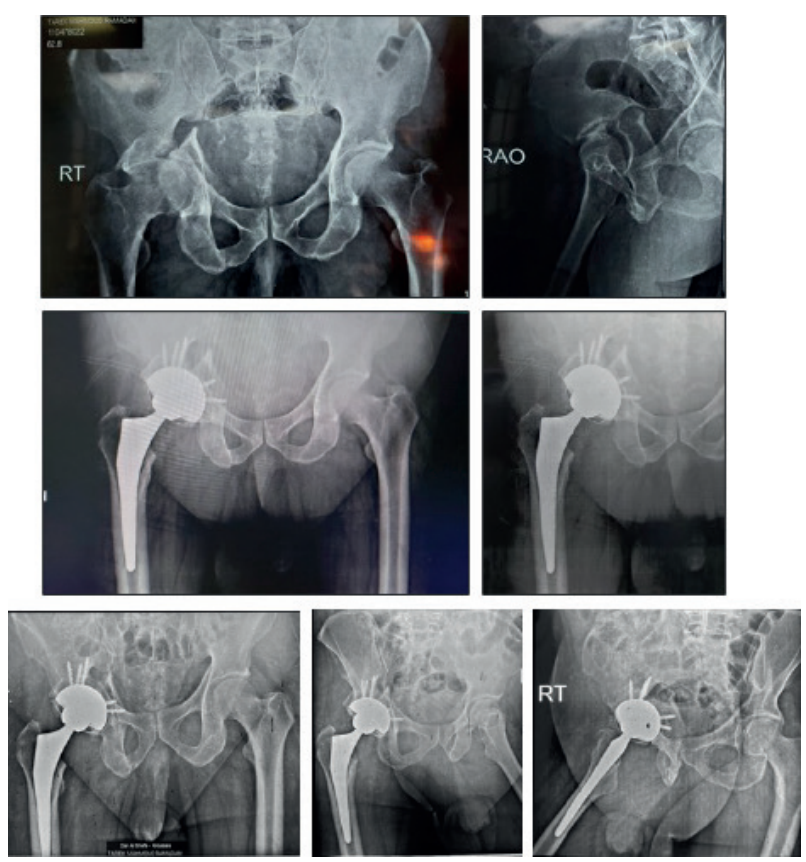

Figure 4. - A case of neglected transverse and posterior wall acetabular fracture with immediate postoperative $\mathrm{X}$ ray and latest $\mathrm{X}$ ray follow up AP, obturator oblique and iliac oblique views showing stable implant and union of the fracture.

described by De Lee and Charnley (12). Acetabular hip center, and migration of acetabular component were considered after the method proposed by Callaghan et al (13). The vertical distance from the center of femoral head to the inter-teardrop line and the horizontal distance to the perpendicular to this line at the teardrop figure were calculated. A normal hip center is reported to be 12 to $14 \mathrm{~mm}$ above the inter-teardrop line and 33 to $43 \mathrm{~mm}$ lateral to the acetabular teardrop (14). A high hip center was arbitrarily defined as having the center of rotation on an anteroposterior radiograph greater than $35 \mathrm{~mm}$ proximal to the inter-teardrop line (15). A component was described as radiographically unstable if a $1 \mathrm{~mm}$ or greater lucent line occurred across all 3 acetabular zones or if any measurable cup migration occurred (16). Loosening was also characterized by a change in the component abduction angle of greater than $10^{\circ}$ or in the horizontal or vertical position of greater than $6 \mathrm{~mm}$ observed in successive radiographs, after correcting for magnification (15). Radiological signs of bony non-union were also checked (16).

\section{RESULTS}

Radiological results at latest follow up and by comparing serial radiographs showed that all cases had a well-fixed implant without evidence of loosening or mal orientation. Hip center was restored in these cases.

The average preoperative Harris hip rating was 46 points, which is attributed to the nature of cases and delay in presentation, and the average last postoperative rating was 84 points. 5 patients had an excellent score (90 to 100) and 2 had good score (80 to 90). No infection, neurological complications were reported. Acceptable leg length equality was achieved in all cases.

\section{DISCUSSION}

In this series of neglected acetabular fracture cases that underwent THA, we applied the technique of acetabular distraction prior to insertion of porous metal cups to perform acetabular reconstruction. At a mean of 18 months' follow-up (range, 12 to 36 months), all the patients showed a significant clinical improvement post-operatively, and none of the patients needed revision.

Management of neglected residually displaced acetabular fractures may present a big challenge. Conservative treatment cannot often achieve stable concentric reduction in presence of unreduced fracture. This will lead to instability and fibrous on growth over the fracture ends. ORIF may not achieve satisfactory results due to the existing bone loss and/or osteoarthritis. Total hip replacement is usually kept in mind as a method of choice, although being technically difficult $(2,3,17)$.

The acetabular distraction technique is a relatively novel approach for managing pelvic discontinuities that was first introduced by Sporer et al in 2012 (7). While the classic THA technique for reconstructing neglected acetabular fractures utilize screws and plates to compress nonunion and achieve mechanical fixation before inserting the acetabular cup, the proposed basis of acetabular distraction is to address acetabular discontinuity with distraction by expanding the defect and creating elastic recoil forces to compress the porous metal construct and 
achieve a stable construct (7-10). The porous metal (Tantalum) acetabular components were used especially in this technique, due to its reported excellent biologic fixation via bony ingrowth and its long- term implants' stability (18-20) in presence of acetabular defects.

With this distraction arthroplasty method, pelvic stability is obtained via fixing the superior and inferior hemipelvis to a highly porous metal cup and/or augment with screws, thereby unitizing the superior and inferior aspects of the pelvis without the necessity to achieve bone healing along the discontinuity/fracture site. The cup acts as a segmental replacement of the acetabulum, with healing occurring up to the cup (or augments if needed) on the superior and inferior borders of the discontinuity, resulting in a unitized and stable hemipelvis (21).

The classic reports that utilize compression plating of the acetabular columns to reduce and stabilize the pelvis in an attempt to create a solid bony substrate for acetabular reconstruction have shown a potential disadvantage in the form of larger surgical exposure and soft tissue stripping that may lead to increased infection rates and decreased osteogenic healing potential of the remaining host bone $(22,23)$. In this proposed technique that does not necessitate extensive surgical exposure and soft tissue stripping, this technique may provide initial stability and adequate fixation without hindering proper osseointegration to occur. The initial stability depends on the strong pelvic 'recoil' around a cup as a result of ligamentotaxis from the stout pelvic ligaments, as well as the inherent material properties of the implant, in the form of high coefficient of friction that provides excellent initial stability. On the other hand, Trabecular metal, or tantalum, is an ideal choice to allow for bone ingrowth and longterm fixation due to the high porosity and excellent strength $(18-20,24)$.

The study has several limitations: First, the absence of a control group makes it difficult to make a conclusion regarding the impact of this technique compared to other techniques. Second, the series is small and the follow up is short. Ideally, a larger series and longer follow up is needed to better judge the efficacy of this technique and document its longevity. Also, the absence of selection criteria for the patients who underwent the procedure presents a source of bias, which should affect the evidence presented.

\section{CONCLUSION}

Neglected or untreated acetabular fractures with or without femoral head dislocation can represent a challenging surgical situation. This scenario may closely resemble a revision setting with a combined segmental and cavitary acetabular defects with over-added difficulty in restoring the hip center and achieving stable reconstruction. This study applies a treatment method for these complex cases using porous metal components through a distraction technique with good early clinical and radiological outcomes. More studies using larger cohort with longer follow up are needed for better evaluation of the success of this technique.

\section{REFERENCES}

1. Morison Z, et al. Total hip arthroplasty after acetabular fracture is associated with lower survivorship and more complications. Clin Orthop Relat Res. 2016 ; 474 : 392-8.

2. Letournel E. Acetabulum fractures: classification and management. Orthop. Trauma Dir. 2007 ; 5 : 27-33.

3. Mears DC, Velyvis JH, Chang C-P. Displaced acetabular fractures managed operatively : indicators of outcome. Clin Orthop Relat Res. 2003 ; 407 : 173-186.

4. Morita M, Seki T, Takegami Y, Kasai T, Higuchi Y, Ishiguro N. Acetabular fracture non-union with pelvic discontinuity treated with two-stage total hip arthroplasty after intraand extra-articular plate fixation. Nagoya J. Med. Sci. 2019 ; 81 : 113-119.

5. Bhende H. Total hip replacement following acetabular fractures. Indian J Orthop [serial online] 2002 [cited 2012 Nov 16] ; $36: 33-5$.

6. Harris WH. Traumatic arthritis of the hip after dislocation and acetabular fractures : treatment by mold arthroplasty. An end-result study using a new method of result evaluation. J Bone Joint Surg Am 1969 ; 51 : 737.

7. Sporer SM, Bottros JJ, Hulst JB, et al. Acetabular distraction: an alternative for severe defects with chronic pelvic discontinuity? Clin.Orthop.Rela.Res. 2012; 470 : 3156-63.

8. Sheth NP, Melnic CM, Brown N, et al. Two-centre radiological survivorship of acetabular distraction technique for treatment of chronic pelvic discontinuity: mean five-year follow-up. J.Bone Joint Surg. 2018 ; 90914. 
9. Pitkin M. Distraction Implantation. A New Technique in Total Joint Arthroplasty and Direct Skeletal Attachment. EC Orthop 2018 May ; 9 : 285-292.

10. Brown NM, Hellman M, Haughom BH, Shah RP, Sporer SM, Paprosky WG. Acetabular distraction : an alternative approach to pelvic discontinuity in failed total hip replacement. J Bone Joint Surg Br. 2014 ; 96 : 73-77.

11. Garbuz D, Morsi E, Mohamed N, et al. Classification and reconstruction in revision acetabular arthroplasty with bone stock deficiency. Clin Orthop Relat Res 1996 ; 98.

12. DeLee JG, Charnley J. Radiological demarcation of cemented sockets in total hip replacement. Clin Orthop Relat Res 1976 ; 20.

13. Callaghan JJ, Salvati EA, Pellicci PM, et al. Results of revision for mechanical failure after cemented total hip replacement, 1979 to 1982 . A two to five-year follow-up. $J$ Bone Joint Surg Am 1985; 67 : 1074.

14. Brown EC, Kelley SS. Determination of the hip center. In : Bono JV, McCarthy JC, Thornhill TS, et al, editors. Revision total hip arthroplasty. New York: SpringerVerlag ; 1999 ; p. 320.

15. Russotti GM, Harris WH. Proximal placement of the acetabular component in total hip arthroplasty. A longterm follow-up study. J Bone Joint Surg Am 1991 ; 73 : 587.

16. Weeden SH, Schmidt RH. The use of tantalum porous metal implants for Paprosky $3 \mathrm{~A}$ and $3 \mathrm{~B}$ defects. $J$ Arthroplasty 2007 ; 22(6 Suppl 2) : 151.

17. Garrett JC, Epstein HC, Harris WH, Harvey JP Jr, Nickel VL. Treatment of unreduced traumatic posterior dislocations of the hip. J Bone Joint Surg Am 1979; 61 : 2-6.

18. Sporer SM, Paprosky WG. Acetabular revision using a trabecular metal acetabular component for severe acetabular bone loss associated with a pelvic discontinuity. J Arthroplasty 2006 ; 21(6 Suppl 2) : 87.

19. Mahmoud AN, Sundberg M, Flivik G. Comparable Results with Porous Metal Augments in Combination with Either Cemented or Uncemented Cups in Revision Hip Arthroplasty: An Analysis of One Hundred FortySeven Revisions at a Mean of Five Years. The Journal of Arthroplasty 2017 ; 32 : 1612-1617.

20. Batuyong, ED, Brock HS, Thiruvengadam N, Maloney WJ, Goodman SB, Huddleston JI. Outcome of porous tantalum acetabular components for Paprosky type 3 and 4 acetabular defects. J Arthroplasty. 2014 ; 29 : 1318-1322.

21. Abdel MP et al. Pelvic Discontinuity Associated with Total Hip Arthroplasty: Evaluation and Management. $J$ Am Acad Orthop Surg 2017 ; 25 : 330-338.

22. Stiehl JB, Saluja R, Diener T. Reconstruction of major column defects and pelvic discontinuity in revision total hip arthroplasty. J Arthroplasty. 2000 ; 15 : 849-857.

23. Udomkiat P, Dorr LD, Won YY, Longjohn D, Wan Z. Technical factors for success with metal ring acetabular reconstruction. J Arthroplasty. 2001 ; 16 : 961-969.

24. Bobyn JD, Stackpool GJ, Hacking SA, Tanzer M, Krygier JJ. Characteristics of bone ingrowth and interface mechanics of a new porous tantalum biomaterial. $J$ Bone Joint Surg [Br] 1999 ; 81-B : 907-914. 